\title{
Provenance and sediment characteristics of contemporary gravel deposits at Sellicks Beach, eastern shore of Gulf St Vincent, South Australia
}

\author{
J. H. CANN ${ }^{1 *}$, C. S. LOWER ${ }^{1,2}$ AND J. B. JAGO ${ }^{1}$
}

${ }^{1}$ School of Natural and Built Environments and the Barbara Hardy Institute, University of South Australia, Mawson Lakes, South Australia 5095, Australia. ${ }^{2}$ BHP Billiton, 55 Grenfell Street, Adelaide, South Australia 5000, Australia. *Corresponding author: john.cann@unisa.edu.au

\section{SUPPLEMENTARY PAPERS}

\author{
Australian Journal of Earth Sciences (2014) 61, \\ http://dx.doi.org/10.1080/08120099.2014.941400
}

Copies of Supplementary Papers may be obtained from the Geological Society of Australia's website (www.gsa.org.au), the Australian Journal of Earth Scienecs website (www.ajes.com.au) or from the National Library of Australia's Pandora archive (http://nla.gov.au/nla.arc-25194).

\section{SUPPLEMENTARY PAPERS}

Table 1 Numerical data for selected clasts at Site \#4, Cactus Canyon, Sellicks Beach. GPS 35²0'29.64"S, 138²6'16.41"E.

Table 2 Numerical data for selected clasts at Site \#3, Sellicks Beach. GPS $35^{\circ} 20^{\prime} 19.56^{\prime \prime}$ S, $138^{\circ} 26^{\prime} 21.34^{\prime \prime} \mathrm{E}$.

Table 3 Numerical data for selected clasts at Site \#2, Sellicks Beach. GPS 35²0'10.33"S, 138²6'26.14”E.

Table 4 Numerical data for selected clasts at Site \#1, Boat ramp, Sellicks Beach. GPS 35¹9'48.63"S, $138^{\circ} 26^{\prime} 29.98^{\prime \prime} \mathrm{E}$. 
Table 1 Numerical data for selected clasts at Site \#4, Cactus Canyon, Sellicks Beach. GPS 35²0'29.64"S, $138^{\circ} 26^{\prime} 16.41^{\prime \prime E}$

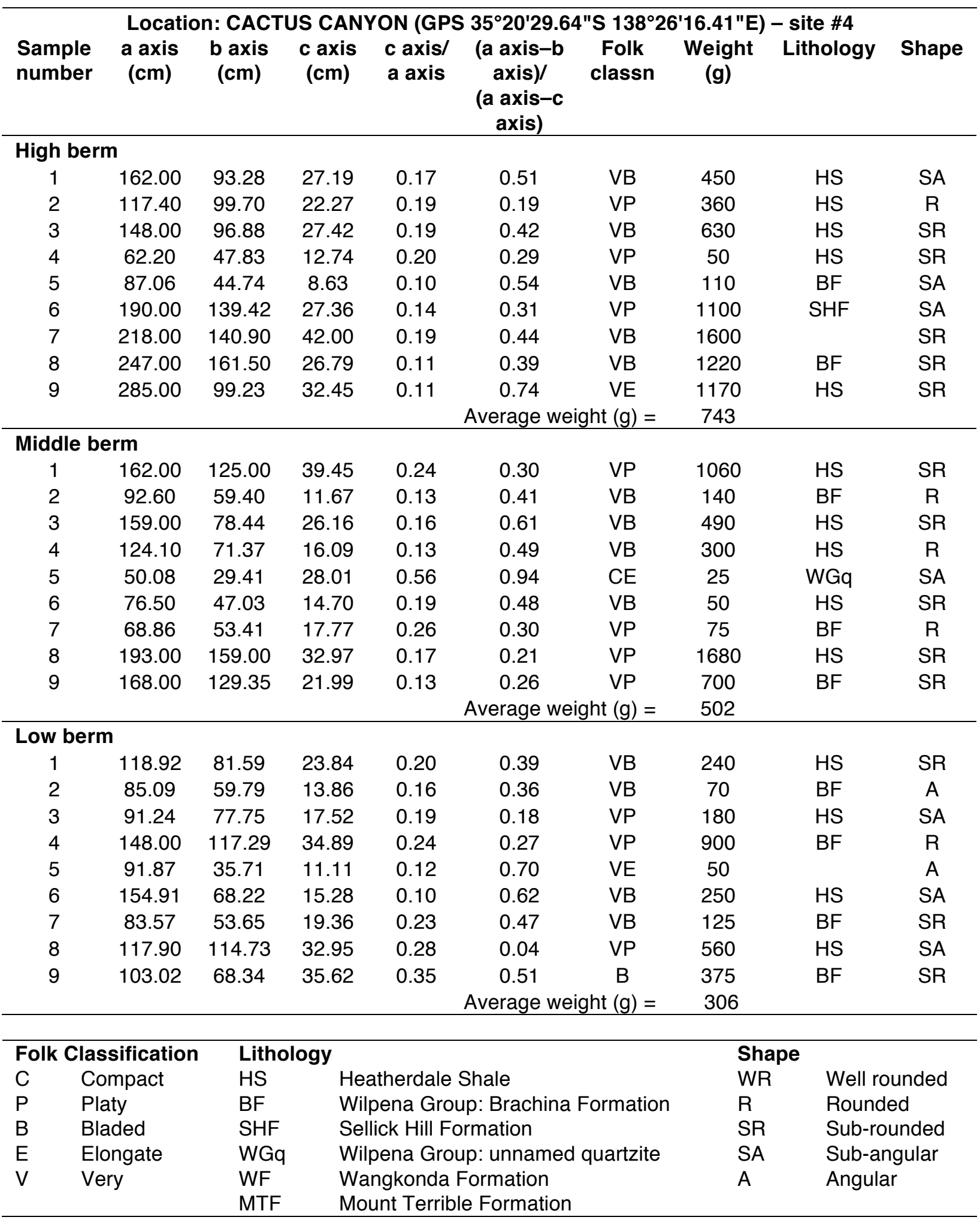


Table 2 Numerical data for selected clasts at Site \#3, Sellicks Beach. GPS 35²0'19.56"S, 138²6'21.34"E.

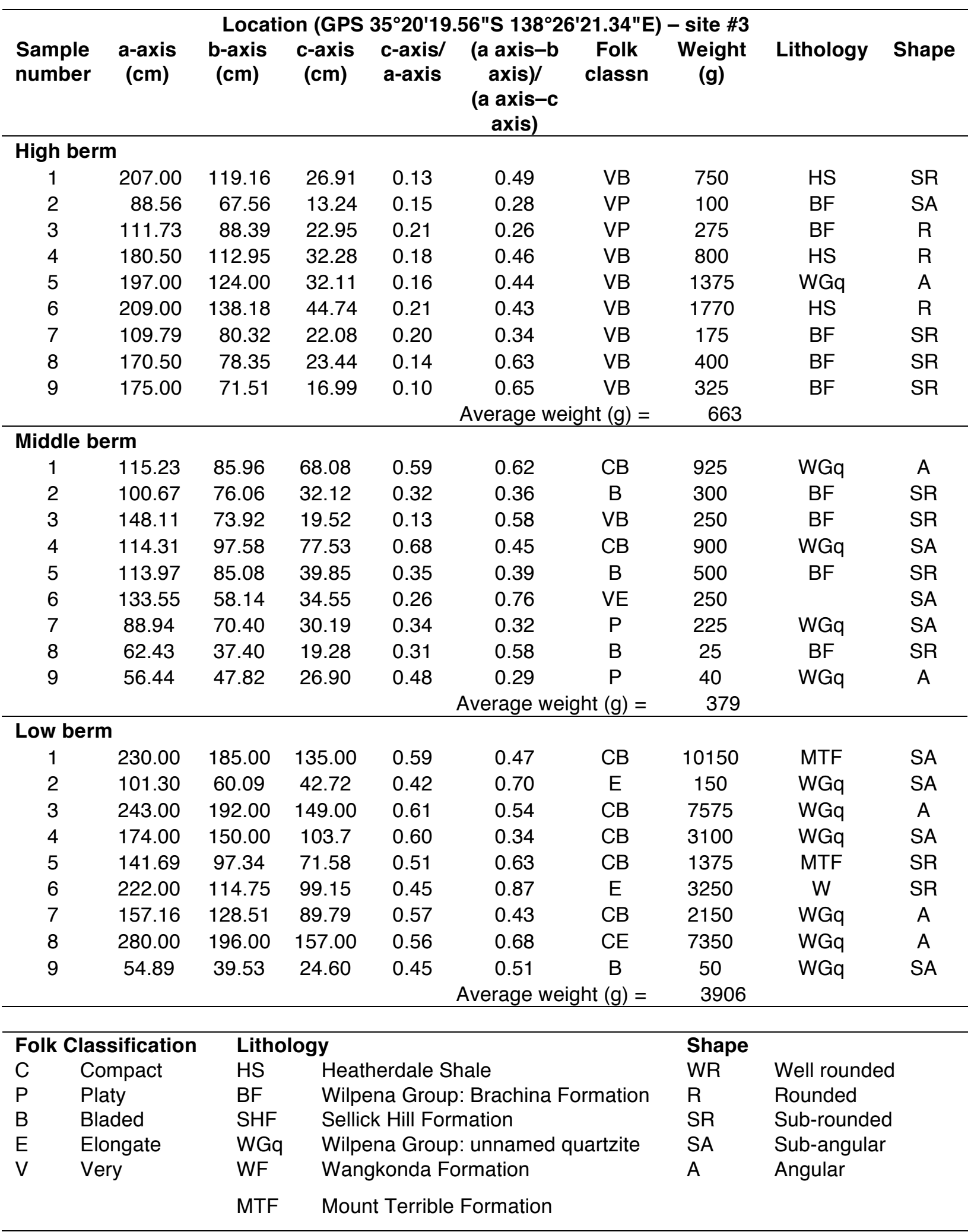


Table 3 Numerical data for selected clasts at Site \#2, Sellicks Beach. GPS $35^{\circ} 20^{\prime} 10.33^{\prime \prime} \mathrm{S}, 138^{\circ} 26^{\prime} 26.14^{\prime \prime} \mathrm{E}$.

\begin{tabular}{|c|c|c|c|c|c|c|c|c|c|}
\hline \multirow[b]{2}{*}{$\begin{array}{l}\text { Sample } \\
\text { number }\end{array}$} & \multicolumn{7}{|c|}{ Location: (GPS $35^{\circ} 20^{\prime} 10.33^{\prime \prime S ~} 138^{\circ} 26^{\prime} 26.14^{\prime E}$ ) - site \#2 } & \multirow[b]{2}{*}{ Lithology } & \multirow[b]{2}{*}{ Shape } \\
\hline & $\begin{array}{l}\text { a-axis } \\
(\mathrm{cm})\end{array}$ & $\begin{array}{l}\text { b-axis } \\
(\mathrm{cm})\end{array}$ & $\begin{array}{l}\text { c-axis } \\
(\mathrm{cm})\end{array}$ & $\begin{array}{l}\text { c-axis/ } \\
\text { a-axis }\end{array}$ & $\begin{array}{c}\text { (a axis-b } \\
\text { axis)/ } \\
(a \text { axis-c } \\
\text { axis) }\end{array}$ & $\begin{array}{l}\text { Folk } \\
\text { classn }\end{array}$ & $\begin{array}{l}\text { Weight } \\
\text { (g) }\end{array}$ & & \\
\hline \multicolumn{10}{|c|}{ High berm } \\
\hline 1 & 62.69 & 51.66 & 23.74 & 0.38 & 0.28 & $P$ & 110 & WGq & SR \\
\hline 2 & 55.88 & 42.68 & 17.78 & 0.32 & 0.35 & B & 80 & WGq & SR \\
\hline 3 & 46.57 & 32.25 & 20.20 & 0.43 & 0.54 & B & 50 & $\mathrm{BF}$ & SR \\
\hline 4 & 44.33 & 29.21 & 14.20 & 0.32 & 0.50 & B & 30 & WGq & SA \\
\hline 5 & 68.76 & 51.09 & 20.18 & 0.29 & 0.36 & VB & 120 & $\mathrm{BF}$ & SR \\
\hline 6 & 71.75 & 47.42 & 30.46 & 0.42 & 0.59 & $\mathrm{~B}$ & 100 & $\mathrm{BF}$ & SA \\
\hline 7 & 70.06 & 38.05 & 18.78 & 0.27 & 0.62 & VB & 90 & WGq & SR \\
\hline 8 & 55.80 & 35.43 & 24.18 & 0.43 & 0.64 & $\mathrm{~B}$ & 70 & WGq & SA \\
\hline \multirow[t]{2}{*}{9} & 31.71 & 28.87 & 28.46 & 0.90 & 0.87 & C & 30 & & SA \\
\hline & & & & & \multicolumn{2}{|c|}{ Average weight $(\mathrm{g})=$} & 76 & & \\
\hline \multicolumn{10}{|c|}{ Middle berm } \\
\hline 1 & 77.61 & 66.37 & 59.85 & 0.77 & 0.63 & C & 350 & WGq & SA \\
\hline 2 & 65.17 & 38.00 & 29.46 & 0.45 & 0.76 & $\mathrm{E}$ & 120 & WGq & SR \\
\hline 3 & 102.76 & 70.87 & 46.62 & 0.45 & 0.57 & B & 400 & BF & SR \\
\hline 4 & 60.17 & 51.11 & 36.25 & 0.60 & 0.38 & CB & 120 & WGq & SA \\
\hline 5 & 68.49 & 41.45 & 16.40 & 0.24 & 0.52 & VB & 110 & BF & SR \\
\hline 6 & 69.41 & 44.80 & 32.29 & 0.47 & 0.66 & B & 200 & WGq & SA \\
\hline 7 & 59.08 & 43.24 & 39.06 & 0.66 & 0.79 & CE & 120 & WGq & SA \\
\hline 8 & 115.32 & 86.75 & 54.89 & 0.48 & 0.47 & $B$ & 700 & $\mathrm{BF}$ & SR \\
\hline \multirow[t]{2}{*}{9} & 101.37 & 93.95 & 34.32 & 0.34 & 0.11 & $P$ & 450 & & SR \\
\hline & & & & & \multicolumn{2}{|c|}{ Average weight $(\mathrm{g})=$} & 286 & & \\
\hline \multicolumn{10}{|c|}{ Low berm } \\
\hline 1 & 250.00 & 200.00 & 150.00 & 0.60 & 0.50 & CB & 5600 & WGq & A \\
\hline 2 & 170.00 & 80.90 & 55.59 & 0.33 & 0.78 & $E$ & 1007 & WGq & SR \\
\hline 3 & 58.34 & 44.45 & 31.87 & 0.55 & 0.52 & CB & 250 & $\mathrm{BF}$ & SR \\
\hline 4 & 79.52 & 67.82 & 33.01 & 0.42 & 0.25 & $\mathrm{P}$ & 300 & BF & $\mathrm{R}$ \\
\hline 5 & 260.00 & 42.79 & 31.22 & 0.12 & 0.95 & VE & 2500 & WGq & SR \\
\hline 6 & 215.00 & 41.29 & 32.39 & 0.15 & 0.95 & VE & 2000 & WGq & SA \\
\hline 7 & 109.46 & 84.24 & 38.17 & 0.35 & 0.35 & B & 570 & & SR \\
\hline 8 & 73.78 & 55.24 & 26.00 & 0.35 & 0.39 & B & 200 & BF & SR \\
\hline \multirow[t]{2}{*}{9} & 138.47 & 93.25 & 53.44 & 0.39 & 0.53 & B & 1350 & WGq & SA \\
\hline & & & & & \multicolumn{2}{|c|}{ Average weight $(\mathrm{g})=$} & 1531 & & \\
\hline \multicolumn{2}{|c|}{ Folk Classification } & \multicolumn{3}{|c|}{ Lithology } & \multicolumn{4}{|c|}{ Shape } & \\
\hline C & Compact & \multicolumn{2}{|c|}{ HS } & \multicolumn{2}{|c|}{ Heatherdale Shale } & & WR & \multicolumn{2}{|c|}{ Well rounded } \\
\hline $\mathrm{P}$ & Platy & \multicolumn{2}{|l|}{ BF } & \multirow{2}{*}{\multicolumn{3}{|c|}{ Wilpena Group: Brachina Formation }} & $\mathrm{R}$ & Rounde & \\
\hline $\mathrm{B}$ & aded & SHF & & & & & SR & Sub-rou & ded \\
\hline$E$ & ongate & MG & & Vilpena $\mathrm{G}$ & oup: unname & d quartzite & SA & Sub-ang & ular \\
\hline $\mathrm{V}$ & ry & WF & & Vangkond & a Formation & & $A$ & Angular & \\
\hline & & MTF & & lount Terr & ible Formatior & & & & \\
\hline
\end{tabular}


Table 4 Numerical data for selected clasts at Site \#1, Boat ramp, Sellicks Beach.

GPS 35॰19'48.63"S, $138^{\circ} 26^{\prime} 29.98^{\prime \prime} \mathrm{E}$.

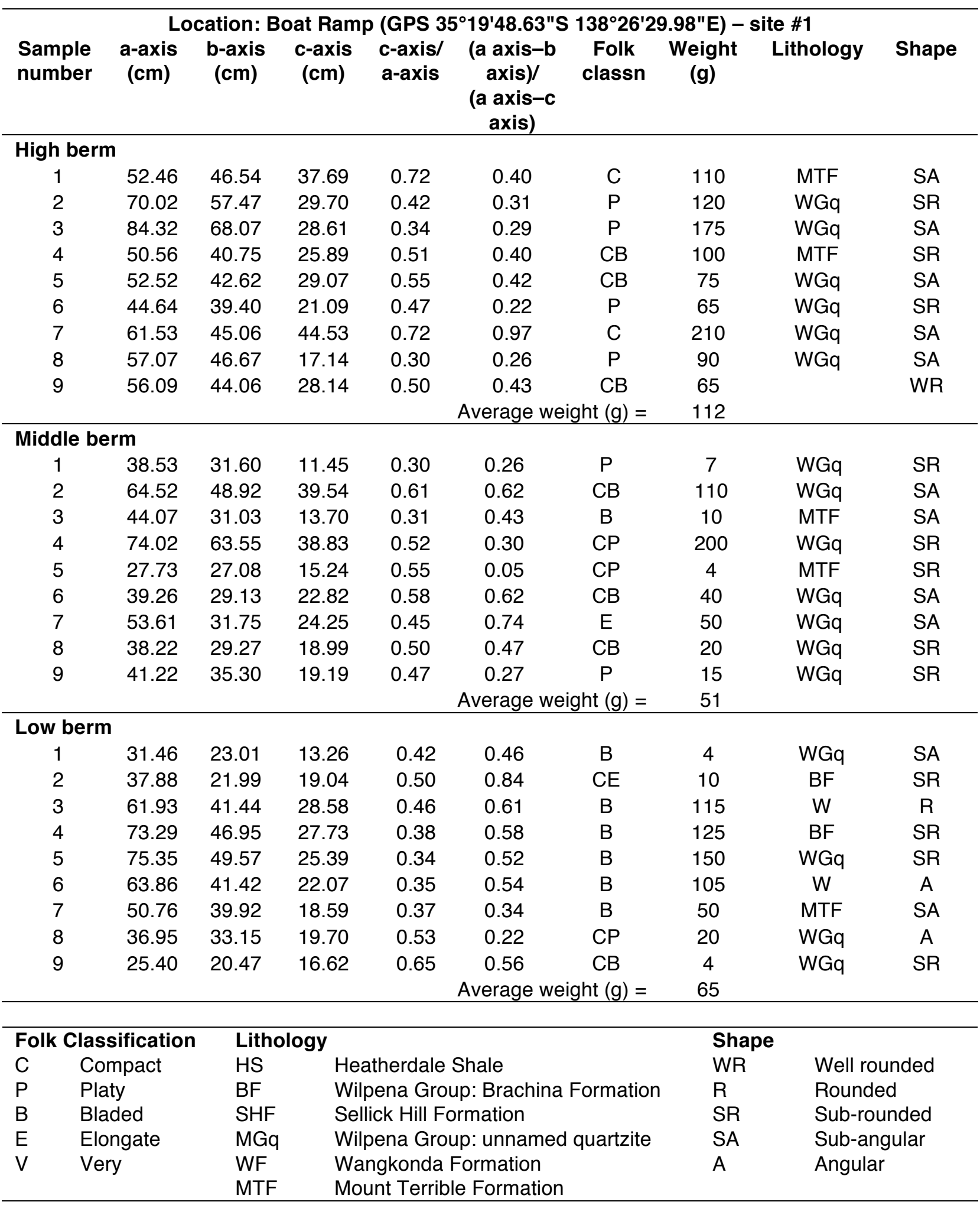

